stitute of Physics, POB 364, 41001 Zagreb, Yugoslavia.

${ }^{1}$ W. C. Stwalley, in Energy, Structure and Reactivity, edited by D. W. Smith and W. B. McRae (Wiley, New York, 1973), p. 259.

${ }^{2}$ R. J. LeRoy, Mol. Spectros. 1, 113 (1973).

${ }^{3}$ W. C. Stwalley, Contemp. Phys. 19, 65 (1978).
${ }^{4}$ M. Movre and G. Pichler, J. Phys. B $\underline{10}, 2631$ (1977).

${ }^{5}$ K. Niemax and G. Pichler, J. Phys. B $\underline{8}, 179$ (1975).

${ }^{6}$ P. Kusch and M. M. Hessel, J. Chem. Phys. 68 , 2591 (1978).

${ }^{7}$ P. R. Fontana, Phys. Rev. 125, 1597 (1962).

\title{
Inner-Shell Ionization Induced by Nuclear Coulomb Excitation in Collisions of Very Heavy Ions
}

\author{
Gerhard Soff \\ Gesellschaft für Schwerionenforschung, 6100 Darmstadt, Germany \\ and \\ Volker Oberacker, and Walter Greiner \\ Institut für Theoretische Physik, Johann Wolfgang Goethe Universität, 6000 Frankfurt am Main, Germany \\ (Received 19 June 1978)

\begin{abstract}
$K$ - and $L$-shell ionization of ${ }^{238} \mathrm{U}$ with Xe and $\mathrm{U}$ projectiles is investigated. Internal conversion following nuclear Coulomb excitation which is particularly important for deformed result of internal conversion, about $0.1-0.3 \mathrm{~K}$ holes per central collision are created.
\end{abstract} \\ heavy nuclei is compared with direct ionization of inner-shell electrons in superheavy \\ quasimolecules. Both processes exhibit different impact-parameter dependences. As a
}

In collisions of deformed heavy nuclei with incident energies slightly below the interaction barrier, large Coulomb excitation probabilities are achieved. They give rise to a strong background for direct inner-shell ionization, ${ }^{1-8}$ and for the photon spectrum of the superheavy quasimolecule. ${ }^{9-11}$ In the ${ }^{208} \mathrm{Pd}-{ }^{208} \mathrm{~Pb}$ system the background effects are negligible ${ }^{4}$ because the highlying first excited states of the doubly magic nucleus ( $3^{-}$at $2.615 \mathrm{MeV}$ and $2^{+}$at $4.086 \mathrm{MeV}$ ) are populated rather weakly. On the other hand, soft nuclei like the actinides exhibit a quite different nuclear spectrum (several rotational bands with high level density).

In the following we outline the theoretical description of inner-shell ionization via internal conversion and present numerical results for
${ }^{136} \mathrm{Xe}-{ }^{238} \mathrm{U}$ and ${ }^{238} \mathrm{U}-{ }^{238} \mathrm{U}$ collisions. The number of created vacancies $P$ per collision caused by a nuclear transition of an initial state $n$ to a final state $m$ is given by

$$
P(n \rightarrow m)=P_{n}^{\text {occ }} W^{e^{-}}(n \rightarrow m) .
$$

The threshold condition reads $E_{n}-E_{m}>E_{b}$, where $E_{b}$ is the binding energy of the electron considered. $P_{n}{ }^{\text {occ }}$ denotes the occupation probability of the state $n$. We emphasize that in general $P_{n}$ occ differs from the Coulomb excitation probability $P_{n}{ }^{\text {exc }}$ of the corresponding nuclear level. There is an additional population due to $\gamma$ and conversion-electron cascades following the excitation of states $i$ with energies $E_{i}>E_{n}$. For $K$-shell conversion the branching ratio $W_{K} e^{-}$is defined by

$$
W_{K}{ }^{-}(n \rightarrow m)=\frac{T_{K}{ }^{-}(n \rightarrow m) U_{K}(n)}{\sum_{k}\left[T^{\gamma}(n \rightarrow k)+T_{k}{ }^{e^{-}}(n \rightarrow k) U_{K}(n)+T_{L}{ }^{e^{-}}(n \rightarrow k) U_{L}(n)+\cdots+T^{\left.e^{+e^{-}}(n \rightarrow k)\right]}\right.} .
$$

$T^{\gamma}, T^{e^{-}}$, and $T^{e^{+} e^{-}}$are the transition rates due to $\gamma$ decay, internal conversion, and internal pair formation. Since $T^{e^{+} e^{-}} \approx 10^{-4} T^{\gamma}$, we can neglect $T^{e^{+} e^{-}}$in the denominator of Eq. (2). The factor

$$
U(n)=\left[N-\sum_{i=1}^{n-1} \sum_{k} P(i \rightarrow k)\right] / N, \quad 0 \leqslant U(n) \leqslant 1,
$$

accounts for electron-number conservation, reflecting the fact that internal conversion cannot take place if the electron shell has been completely ionized. $N$ denotes the occupation number of the electron shell, i.e., $N_{K}=2$ and $N_{L}=8$. The small conversion probabilities for higher-state transitions allow us to insert $U(n)=1$, which modifies inconsiderably the obtained results. Employing the defini- 
tion of the conversion coefficient

$$
\alpha=T^{e^{-}} / T^{\gamma}
$$

we obtain

$$
W^{e^{-}}(n \rightarrow m)=\alpha(n \rightarrow m) U(n) W^{\gamma}(n \rightarrow m),
$$

with

$$
W^{\gamma}(n \rightarrow m)=\frac{T^{\gamma}(n \rightarrow m)}{\sum_{k} T^{\gamma}(n \rightarrow k)\left[1+\alpha_{K}(n \rightarrow k) U_{K}(n)+\alpha_{L}(n \rightarrow k) U_{L}(n)+\cdots\right]} .
$$

The Coulomb excitation probabilities $P_{n}{ }^{\text {exc }}$ of collective levels in ${ }^{238} \mathrm{U}$ are computed with an extended semiclassical coupled-channels code. A detailed discussion of the results can be found in Oberacker and Soff. ${ }^{12}$ Excitation and photon emission are dominated by $E 2$ transitions. We evaluate the collective quadrupole matrix elements $\langle m\|M(E 2)\| n\rangle$ in the framework of the rotation-vibrational model. ${ }^{13}$ The coefficients $\alpha$ for conversion of bound-state electrons, which depend on nuclear charge $Z$, transition energy $\Delta E=E_{n}-E_{m}$, and multipolarity, are taken from Hager and Seltzer. ${ }^{14}$

Combining Eqs. (1) and (5) we can write

$$
P(n \rightarrow m)=P^{\gamma}(n \rightarrow m) \alpha(n \rightarrow m) U(n),
$$

with the photon-emission probability $P^{\gamma}(n \rightarrow m)=P_{n}{ }^{\text {occ }} W^{\gamma}(n \rightarrow m)$. We still need the occupation probability $P_{n}{ }^{\text {occ }}$ of the decaying level at the time when internal conversion takes place. It is given by the direct excitation probability $P_{n}{ }^{\text {exc }}$ and an additional population due to $\gamma$ and $e^{-}$cascades from higher states. If the nuclear levels are arranged such that the level with highest energy is labeled by $n=1$, we obtain the recursion relation

$$
P_{n}^{\text {occ }}=P_{n}{ }^{\text {exc }}+\sum_{i=1}^{n-1} P_{i}^{\text {occ }} \tilde{W}^{\gamma}(i \rightarrow n)
$$

with

$$
\tilde{W}^{\gamma}(i \rightarrow n)=\frac{T^{\gamma}(i \rightarrow n)\left[1+\alpha_{K}(i \rightarrow n) U_{K}(i)+\alpha_{L}(i \rightarrow n) U_{L}(i)\right]}{\sum_{k} T^{\gamma}(i \rightarrow k)\left[1+\alpha_{K}(i \rightarrow k) U_{K}(i)+\alpha_{L}(i-k) U_{L}(i)\right]} .
$$

Because of spin and parity selection rules, only a small fraction of the levels contributes to the cascase. We are calculating only total probabilities and not angular distributions of emitted electrons. Therefore we would like to stress that the $e^{-}$conversion process depends only on the Coulomb-excitation probabilities $P_{n}{ }^{\text {exc }}$ and not on the corresponding amplitudes $a_{n}{ }^{\text {exc }} \cdot{ }^{15}$ In consequence the $e^{-}$emission can be treated as incoherent.

In the center-of-mass frame the differential cross section for internal conversion with respect to the ion scattering angle is given by

$$
\frac{d \sigma^{e^{-}}}{d \Omega_{\mathrm{c}, \mathrm{m} .}}=\sum_{1,2} \sum_{n_{0}, m} P\left(n \rightarrow m, \theta_{\mathrm{c} . \mathrm{m}_{\bullet}}\right) \frac{d \sigma^{\text {scatt }}\left(\theta_{\mathrm{c}, \mathrm{m}_{\bullet}}\right)}{d \Omega_{\mathrm{c} . \mathrm{m}_{\bullet}}},
$$

where $d \sigma^{\text {scat }}$ denotes the Rutherford cross section. The first sum in Eq. (10) describes conversion in the projectile and in the target. For identical systems one has to symmetrize Eq. (10):

$$
\frac{d \sigma_{\mathrm{sym}} e^{-}}{d \Omega_{\mathrm{c}, \mathrm{m} .}}=2 \sum_{n_{0} m}\left[P\left(n \rightarrow m, \theta_{\left.\mathrm{c}, \mathrm{m}_{0}\right)}\right) \frac{d \sigma^{\text {scatt }}\left(\theta_{\mathrm{c}, \mathrm{m}}\right)}{d \Omega_{\mathrm{c}, \mathrm{m}_{\bullet}}}+P\left(n \rightarrow m, \pi-\theta_{\mathrm{c}, \mathrm{m}_{0}}\right) \frac{d \sigma^{\mathrm{scatt}}\left(\pi-\theta_{\mathrm{c}, \mathrm{m}_{\bullet}}\right)}{d \Omega_{\mathrm{c}, \mathrm{m} .}}\right] .
$$

In this case it follows for the total cross section we have

$$
\sigma_{\text {sym }}=\frac{1}{2} \int \frac{d \sigma_{\text {sym }}}{d \Omega_{\text {c.m. }}} d \Omega_{\text {c.m. }} .
$$

The symmetrization of the cross section has been performed classically. In a quantum-mechanical treatment an additional interference term arises causing oscillations in the angular distribution. In heavy-ion collisions these oscilla- tions average out completely because of the large Sommerfeld parameter $n(n \approx 600$ for $\mathrm{U}-\mathrm{U})$, and one obtains the classical limit.

For the special case $\alpha=1$ we can compute the nuclear photon spectrum (see, e.g., Müller et $\left.a l .{ }^{16}\right)$, which has to be compared with the quasimolecular spectrum..$^{-11}$ If we replace $\alpha$ by the internal-pair-formation coefficient $\beta$, the cor- 
responding positron production rate can be evaluated. ${ }^{16}$

Figure 1 shows the nuclear photon spectrum for ${ }^{238} \mathrm{U}^{238} \mathrm{U}$ collision, with $E_{1 \mathrm{ab}}=5.39 \mathrm{MeV} / \mathrm{N}$ corresponding to a distance of closest approach $R_{\text {min }}=19 \mathrm{fm}$. The total cross sections for the different nuclear transitions exceed the expected quasimolecular photon spectrum (see, e.g., Ref. 9) by at least two orders of magnitude. Therefore we conclude that, in collisions of actinide nuclei with bombarding energies near the Coulomb barrier, no quasimolecular photon spectroscopy is possible.

The dashed lines in Figs. 2 and 3 show the impact-parameter dependence for the number of created vacancies $P(b)$ due to internal conversion at two different ion energies. For central collisions we obtain typical values of $P_{K}(b=0) \approx 0.3$ and $P_{L}(b=0) \approx 2.5$ per nucleus. For impact parameters $b>10 \mathrm{fm}$ the ionization probability decreases very steeply with an exponential behavior $P(b) \sim \exp (-b / a)$. The falloff constants are found to be $a_{K}(\mathrm{Xe}-\mathrm{U})=3.5, a_{K}(\mathrm{U}-\mathrm{U})=4.3, a_{L}(\mathrm{Xe}-\mathrm{U})=13$, and $a_{L}(\mathrm{U}-\mathrm{U})=15 \mathrm{fm}$ at $R_{\min }=19 \mathrm{fm}$. For the identical system U-U in Fig. 3, projectile and target excitation has been considered. The corresponding cross sections, however, should be

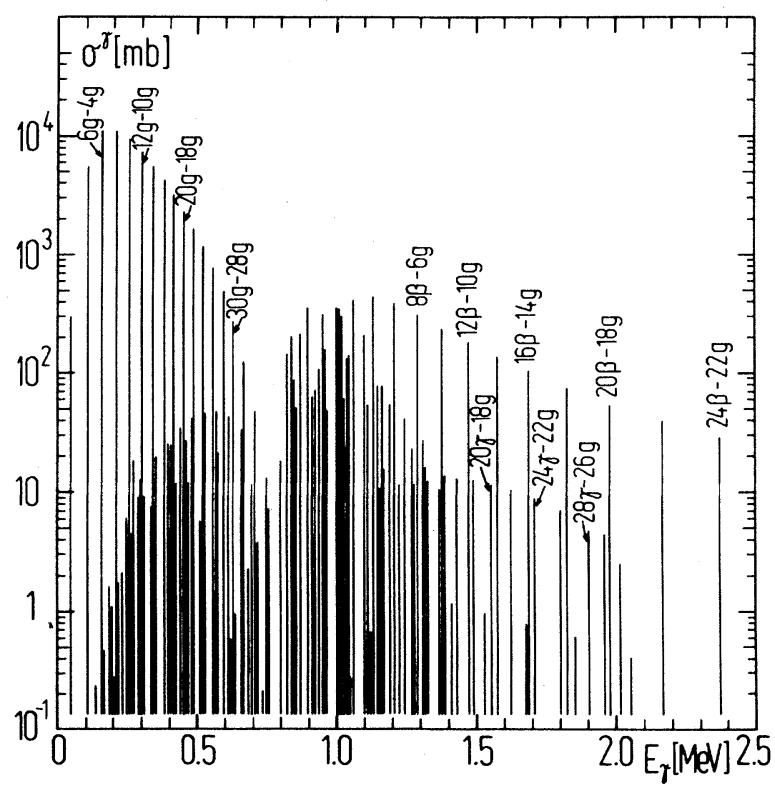

FIG. 1. Total cross sections for photon emission following nuclear Coulomb excitation in a ${ }^{238} \mathrm{U}-{ }^{238} \mathrm{U}$ collision $\left(E_{1 \mathrm{ab}}=5.39 \mathrm{MeV} / N\right)$. Several nuclear transitions between the g.s., $\beta$, and $\gamma$ bands are indicated. Both projectile excitation and target excitation have been considered.
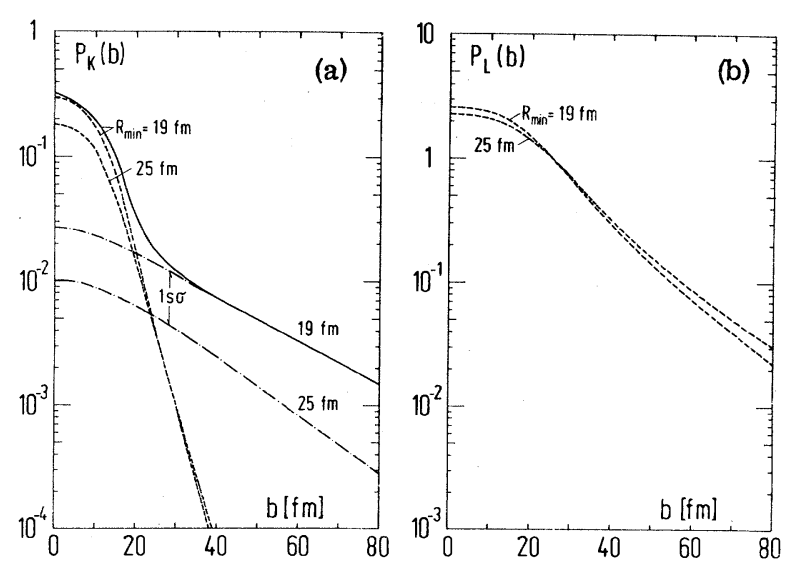

FIG. 2. Number of created vacancies per collision $P(b)$ as a function of impact parameter for (a) $K$-shell and (b) $L$-shell electrons of ${ }^{238} \mathrm{U}$ in ${ }^{136} \mathrm{Xe}^{238} \mathrm{U}$ collisions with $R_{\min }=19$ and $25 \mathrm{fm}$. The contributions from internal conversion following nuclear Coulomb excitation (dashed line) and from direct $1 s \sigma$ ionization of the superheavy quasimolecule (dash-dotted line) are indicated separately. Solid line, incoherent sum of both $K$-vacancy-formation processes for $R_{\mathrm{min}}=19 \mathrm{fm}$.

symmetrized. In central collisions mainly the high spins $I^{\pi} \approx 10^{+}-20^{+}$are excited. When the nucleus cascades down to its ground state, the maximum conversion probability occurs for the $6^{+}-4^{+}, 4^{+}-2^{+}$, and $2^{+}-0^{+}$transitions in the groundstate rotational band.

For comparison, the dashed-dotted and dotted lines in Figs. 2(a) and 3(a) exhibit the impactparameter dependence for direct excitation of
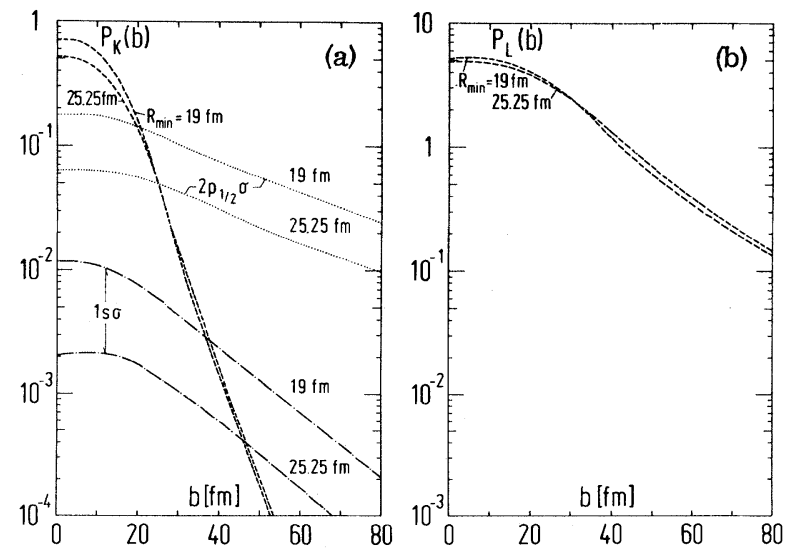

FIG. 3. Same as Fig. 2, but for ${ }^{238} \mathrm{U}-{ }^{238} \mathrm{U}$ collisions with $R_{\mathrm{min}}=19$ and $25.25 \mathrm{fm}$. The dotted lines show the impact-parameter dependence of direct $2 p_{1 / 2} \sigma$ excitation to the continuum, which also contributes to $K$-vacancy formation in uranium. 
the quasimolecular $1 s \sigma$ and $2 p_{1 / 2} \sigma$ states into the upper continuum via radial coupling in the superheavy system. A new aspect compared with earlier calculations ${ }^{1-4}$ is the consideration of the finite nuclear charge distribution according to Soff et al. ${ }^{17}$ The $1 s \sigma$ ionization probability for central collisions is lowered by $21 \%$ for $\mathrm{Xe}-\mathrm{U}$ and by $37 \%$ for $\mathrm{U}-\mathrm{U}\left(R_{\mathrm{min}}=19 \mathrm{fm}\right)$ because of the finite nuclear size. Furthermore, in the U-U system the $1 s \sigma$ state dives into the negative-energy continuum at two center distances $R<33 \mathrm{fm}$. In this case we calculated the bound state by determining the resonance position in the lower continuum and normalizing the corresponding state to 1.

For central collisions the direct ionization probabilities are about one order of magnitude smaller than for the conversion process. At larger impact parameters, on the other hand, the exponential decrease is found to be much weaker: $a_{1 s \sigma}(\mathrm{Xe}-\mathrm{U})=25, a_{1 s \sigma}(\mathrm{U}-\mathrm{U})=17$, and $a_{2 p_{1 / 2} \sigma}(\mathrm{U}-\mathrm{U})=34 \mathrm{fm}$. We have neglected rotational-coupling effects concerning $2 p_{1 / 2} \sigma$ ionization, which may modify the impact-parameter dependence considerably. But the influence of the rotational coupling strongly depends on the number of present vacancies in the $2 p_{3 / 2} \pi$ and $3 p_{3 / 2} \pi$ states brought into the collision, on which no experimental information is available. Therefore we refer to Heiligenthal et al. ${ }^{18}$ for a corresponding model calculation.

The total cross sections for the conversion process turn out to be $\sigma_{K}(\mathrm{Xe}-\mathrm{U})=1.5, \sigma_{\dot{K}}(\mathrm{U}-\mathrm{U})$ $=6.2, \sigma_{L}(\mathrm{Xe}-\mathrm{U})=69$, and $\sigma_{L}(\mathrm{U}-\mathrm{U})=226 \mathrm{~b}$ at $R_{\text {min }}$ $=19 \mathrm{fm}$, where in the U-U system both projectile excitation and target excitation have been considered. In the case of direct ionization, it results in $\sigma_{1 s \sigma}(\mathrm{Xe}-\mathrm{U})=1.5 \mathrm{~b}$, which is comparable with the nuclear process, whereas $\sigma_{1 s \sigma}(\mathrm{U}-\mathrm{U})$ $=0.46$ and $\sigma_{2 p 1 / 2} \sigma(U-U)=21 \mathrm{~b}$. By the Doppler shift of the characteristic $\mathrm{x}$-ray lines, direct ionization $\left(\tau_{\mathrm{dir}} e^{-} \approx 10^{-19} \mathrm{~s}\right)$ can be distinguished experimentally from internal-conversion ionization $\left(\tau_{\text {conv }}{ }^{e^{-}} \approx 10^{-10} \mathrm{~s}\right) .{ }^{19}$ Recent experimental results $^{19,20}$ indeed indicate that both processes are separable.

As an example, for the total number of created $K$ vacancies per collision we present in Fig. 2(a) for the Xe-U system with $R_{\mathrm{min}}=19 \mathrm{fm}$ the incoherent sum (solid line) of both $K$-shell ionization processes. The most striking feature is certainly the two-component distribution with respect to the impact parameter, which should be easily measurable.

We would like to acknowledge enlightening discussions with B. Müller, J. Reinhardt, J. S. Greenberg, and R. Anholt. This work was supported by the Bundesministerium für Forschung und Technologie (BMFT).

${ }^{1}$ W. Betz, B. Muller, G. Soff, and W. Greiner, Phys. Rev. Lett. 37, 1746 (1976).

${ }^{2}$ G. Soff, W. Betz, G. Heiligenthal, J. Kirsch, B. Müller, J. Reinhardt, and W. Greiner, Fizika (Zagreb) 9 , Suppl. 4, 721 (1977).

${ }^{3}$ G. Soff, B. Mưller, and W. Greiner, Phys. Rev. Lett. $\underline{40}, 540$ (1978).

${ }^{4}$ G. Soff, W. Betz, B. Muller, W. Greiner, and

E. Merzbacher, Phys. Lett. $\underline{65 \mathrm{~A}}$, 19 (1978).

${ }^{5}$ J. S. Greenberg, H. Bokemeyer, H. Emling,

E. Grosse, D. Schwalm, and F. Bosch, Phys. Rev. Lett. 39, 1404 (1977).

${ }^{6}$ J. R. McDonald, P. Armbruster, H. H. Behncke, F. Folkmann, S. Hagmann, D. Liesen, P. H. Mokler, and A. Warczak, Z. Phys. A 284, 57 (1978).

${ }^{7}$ R. Anholt and W. E. Meyerhof, Phys. Lett. $64 \mathrm{~A}, 381$ (1978).

${ }^{8}$ R. Anholt, Phys. Rev. A 17, 978 (1978).

${ }^{9} \mathrm{~J}$. Kirsch, W. Betz, J. Reinhardt, G. Soff, B. Mưller, and W. Greiner, Phys. Lett. 72B, 298 (1978).

${ }^{10}$ W. Wölfli, Ch. Stoller, E. Morenzoni, G. Bonani, and M. Stöckli, in Proceedings of an International Symposium on Superheavy Elements, Lubbock, Texas, 1978 (to be published):

${ }^{11}$ W. E. Meyerhof, in Proceedings of the International Symposium on Superheavy Elements, Lubbock, Texas, 1978 (to be published).

${ }^{12}$ V. Oberacker and G. Soff, Z. Naturforsch. $\underline{32 \mathrm{~A}}, 1465$ (1977).

${ }^{13}$ J. M. Eisenberg and W. Greiner, Nuclear Theory

(North-Holland, Amsterdam, 1970), Vol. 1.

${ }^{14}$ R. S. Hager and E. C. Seltzer, Nucl. Data, Sect. A $\underline{4}$, 1 (1968).

${ }^{15}$ Coulomb Excitation, edited by K. Alder and A. Winther (Academic, New York and London, 1966); and V. Oberacker, thesis, Johann Wolfgang Goethe Universität, 1977 (unpublished).

${ }^{16}$ B. Muller, V. Oberacker, J. Reinhardt, G. Soff, W. Greiner, and J. Rafelski, Fizika (Zagreb) 9, Suppl. 4, 765 (1977).

${ }^{17}$ G. Soff, J. Reinhardt, W. Betz, and J. Rafelski, Phys. Scr. 17, 417 (1978).

${ }^{18} \mathrm{G}$. Heiligenthal, W. Betz, G. Soff, B. Muller, and W. Greiner, Z. Phys. A 285, 105 (1978).

${ }^{19} \mathrm{~J}$. S. Greenberg and H. Bokemeyer, private communication, and to be published.

${ }^{20}$ R. Anholft, H.-H. Behncke, S. Hagmann, P. Armbruster, F. Folkmann, and P. Mokler, " $K$-Vacancy Production by $3.6-, 4.75-$, and $5.9-\mathrm{MeV} / \mathrm{amu}{ }^{238} \mathrm{U}$ ions (to be published). 